\title{
Métricas de Valoración de la Gestión del Conocimiento para las Pequeñas y Medianas Empresas del Sector Tecnologías de Información en el Triángulo del Café en Colombia
}

\author{
Diego López ${ }^{(1,2)}$, Carlos E Marulanda ${ }^{(3,4)}, M_{\text {Marcelo López }}^{(1,4)}$ \\ (1) Universidad Nacional de Colombia, Facultad de Administración, Departamento de Informática y \\ computación. Campus La Nubia Manizales-Colombia, (e-mail: dilopezca@unal.edu.co) \\ (2) Universidad de Manizales, Facultad de Ciencias e Ingeniería, carrera 9 \# 19-03, Manizales-Colombia. \\ (3) Universidad Nacional de Colombia, Facultad de Administración, Departamento de Administración. \\ Campus Palogrande. Calle 27 No. 64-60, Manizales-Colombia y \\ (4) Universidad de Caldas, Facultad de Ingeniería, Departamento de Sistemas e Informática, Calle 65 No. \\ 26-10, Manizales-Colombia. (e-mail: carlose@ucaldas,edu.co; mlopez@ucaldas.edu.co)
}

Recibido Oct. 2, 2014; Aceptado Dic. 5, 2014; Versión final recibida Ene. 10, 2014

\begin{abstract}
Resumen
El objetivo del presente trabajo es la presentación de las métricas de valoración de la gestión del conocimiento para las empresas de desarrollo de software del sector tecnologías de información del triángulo del café en Colombia. Se partió de un constructo teórico, se avanzó con la validación estadística a partir del análisis de fiabilidad. Luego se hizo el análisis de correlación y el análisis factorial confirmatorio, con datos de 110 empresas y se complementó con una escala de valoración que permite a cada empresa identificar su estado en el desarrollo de la gestión del conocimiento. Se concluye que la gestión del conocimiento se debe valorar considerando las siguientes categorías: ciclo de vida, tecnologías digitales, cultura organizacional, procesos estratégicos, procesos misionales, procesos de apoyo, competencias personales y relaciones sociales.
\end{abstract}

Palabras clave: gestión del conocimiento, valoración de gestión, evaluación de gestión, tecnologías de la información

\section{Metrics for Assessing Knowledge Management for Small and Medium Size Companies of the Information and Technology Sector in the Colombian Coffee Triangle}

\begin{abstract}
The objective of this work is the presentation of metrics for the evaluation of knowledge management for companies in the software development of the information technology sector in the Colombian coffee triangle. The procedure started from a theoretical construct, advanced with the statistical validation applying analysis of reliability. After that, correlation analysis and confirmatory factor analysis was performed using data from 110 companies and was complemented with a scale of assessment that allows each company identifying its status in the development of knowledge management. It is concluded that the knowledge management must be valued considering the following categories: life cycle, digital technologies, organizational culture, strategic processes, mission processes, support processes, personal competencies and social relations.
\end{abstract}

Keywords: knowledge management, knowledge management, knowledge evaluation, information technologies 


\section{INTRODUCCIÓN}

Dadas las condiciones que deben enfrentar hoy día las organizaciones, en un mercado abierto y muy competitivo, es fundamental para estas, desarrollar estrategias que permitan mejorar su productividad y rentabilidad, en un marco de mejora continua que permita adaptarse con mayor rapidez a las necesidades de los clientes. En este contexto la gestión del conocimiento (GC) aparece como uno de los conceptos de vanguardia más importantes a nivel organizacional y como una disciplina cuyo objetivo se centra en desarrollar el conocimiento en las fases siguientes: adquisición, almacenamiento, transformación, distribución y utilización, con la finalidad de lograr ventajas competitivas (Barney, 1991; Dosi, 1992; Riesco, 2006).

Las PYMES del sector de tecnologías de información ( $\mathrm{TI}$ ) del eje cafetero, no son ajenas a permanecer en una constante vigilancia y desarrollo de nuevas formas de hacer u ofrecer servicios con base en la gestión del conocimiento y en este sentido (Benavides, 1998), (Condo, 2001), (Gallagher, 2001), (Porter M. , 2001), (Limone y Bastidas, 2003), (Castells, 2006), (Folta, 2006), (Joyanes, 2009) y (Sanabria, Morales y Arias, 2010) y (Tallman, 2004), coinciden en la necesidad que las empresas se integren en redes, conglomerados o clúster para solucionar sus problemas de competitividad. Según (Proexport, 2011), el sector de TI en el país es uno de los más promisorios y se caracteriza porque: es tercero en América Latina; es un mercado con un amplio potencial de penetración de las TI; los ingresos del sector se han casi duplicado desde 2005; la industria emplea aproximadamente a 168.000 personas; las principales compañías de software del país ya han sido certificadas en $\mathrm{CMMI}$; se cuenta con: la tasa de piratería más baja de la región, calidad y disponibilidad de recurso humano, los costos de operación altamente competitivos y la infraestructura capaz de soportar operaciones de talla mundial.

En este artículo se presenta la validación y aplicación de un modelo de evaluación de gestión del conocimiento, resultado de la investigación doctoral titulada: desarrollo de modelo de evaluación de gestión del conocimiento para las PYMES del sector TI del triángulo del café de Colombia, la cual partió de las necesidades propias de éstas empresas de la región. El modelo fue validado en 110 empresas desarrolladoras de software, desde sus condiciones particulares y con el apoyo de pruebas estadísticas, a su vez, se complementaron los resultados con la aplicación a una mediana empresa de la región.

En el caso específico de las empresas de software, Sulayman et al. (2012) comentan que las organizaciones modernas de software han empezado a realizar iniciativas de mejora de procesos de software con una visión general de los diferentes enfoques destinados a aumentar la madurez y calidad de sus procesos. En las empresas del presente estudio, es decir, las PYMES de software del triángulo del café, se han identificado algunos factores clave de éxito, como son: el uso de modelos de desarrollo de software, la implantación de programas de mejoramiento, la relación y la cooperación con los clientes, la mejora de procesos, los ciclos cortos, la búsqueda de ayuda financiera externa, la evaluación de procesos, la creación de infraestructuras de apoyo a la comunicación y el compromiso de la gestión, entre otros.

Pawlowsk y Bick (2012) analizaron alrededor de 160 marcos para identificar los factores de éxito y los componentes más importantes de la evaluación de la gestión del conocimiento, e identificaron los siguientes factores críticos de éxito: factores orientados a lo humano, gestión de la organización, la tecnología y la estrategia, los objetivos y la medición. Por otro lado, frente a la evaluación de la gestión del conocimiento Shapira et al. (2006) plantean que la capacidad de conocimiento de las empresas está representado por: cuadros de mando, control de activos intangibles, cuentas de capital y modelos estilizados de conocimiento. Además de incluir el liderazgo, estrategias, alianzas organizativas, o talento (Karadsheh, et al., 2009).

León y Ponjuán (2011), proponen, cuatro dimensiones básicas que conforman el modelo de medición de gestión del conocimiento a saber: actores, procesos estratégicos de la gestión del conocimiento, procesos de apoyo y cultura organizacional, además del sistema de capitales y del sistema de indicadores. Hsun, Chou y Tzeng (2011), sugieren que una infraestructura de conocimiento consiste en la tecnología, la estructura y la cultura, junto con la arquitectura de procesos de conocimiento, para la adquisición, la conversión, la aplicación y la protección del conocimiento como esencia de la capacidad de gestión del conocimiento eficaz de una organización. Con base en estos marcos de referencia, y los planteados por Kaplan y Norton (1996), Sánchez (2003), CEN-3 (2004), Jennex y Olfman (2004), Chang y Wang (2009), Wen (2009), López (2010) y (López et al. (2014), se plantea un modelo teórico de evaluación.

Se define un constructor, que represente el modelo integral de evaluación de gestión del conocimiento, tal como se observa en la figura 1. Un modelo que plantea tres dimensiones de evaluación, en un ciclo permanente (tal como muestran las flechas) como son la dimensión de infraestructura para gestionar conocimiento: que se define a partir de las categorías y variables como sigue en la Tabla 1. En la Tabla 1 Ciclo de vida está referido a las diversas fases para la gestión del conocimiento; Tecnologías digitales son aquellas que configuran un medio natural para el flujo de conocimiento; Cultura organizacional es el 
conjunto de costumbres, hábitos y formas de actuar de las personas y que actúa como un mediador con el conocimiento organizacional.

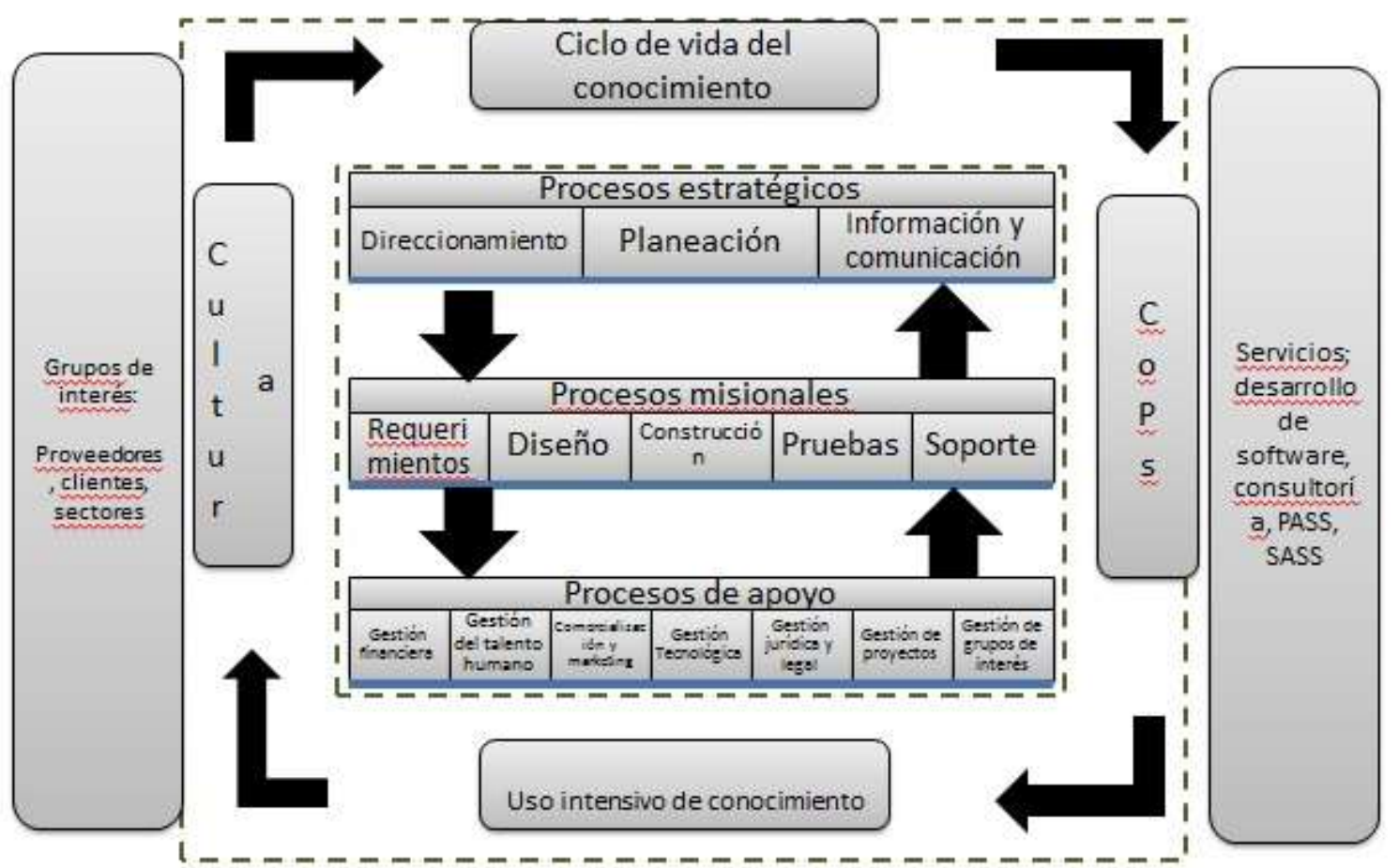

Fig. 1. Modelo de evaluación de GC

Tabla 1: Categorías y variables de la dimensión Infraestructura

\begin{tabular}{|c|c|c|}
\hline Categoría & Variable & Referencias \\
\hline \multirow{6}{*}{ Ciclo de vida } & \multirow{6}{*}{$\begin{array}{l}\text { Identificar } \\
\text { Generar } \\
\text { Retener } \\
\text { Compartir } \\
\text { Aplicar }\end{array}$} & Cameron y Quinn (1999) \\
\hline & & Grant (2002) \\
\hline & & RolMoral \\
\hline & & DelMoral et al. (2007) \\
\hline & & Chen, (2008) \\
\hline & & Kruger y Johnson (2010) \\
\hline \multirow{3}{*}{ Tecnologías digitales } & \multirow{3}{*}{$\begin{array}{l}\text { Básicas } \\
\text { Métodos } \\
\text { Tecnologías de conocimiento }\end{array}$} & (López M. (2010) \\
\hline & & Steinfield et al. (2010) \\
\hline & & Vaccaro et al. (2010), \\
\hline \multirow{5}{*}{ Cultura organizacional } & \multirow{5}{*}{$\begin{array}{l}\text { Visión } \\
\text { Valores } \\
\text { Prácticas } \\
\text { Personas } \\
\text { Narrativa } \\
\text { Sitio de trabajo }\end{array}$} & Chen y Chen, 2011) \\
\hline & & Eftekharzade y Mohammadi 2011) \\
\hline & & Mueller (2013) \\
\hline & & \\
\hline & & \\
\hline
\end{tabular}

La dimensión uso intensivo de conocimiento en procesos organizacionales: que se define a partir de las categorías y variables como sigue en la Tabla 2. En esta Tabla, Procesos estratégicos son aquellos que involucran la dirección de la empresa; Procesos misionales son aquellos que procesan recursos para obtener el servicio conforme al cliente; Procesos de apoyo son aquellos necesarios para satisfacer los requisitos de los clientes internos.

La dimensión comunidades de práctica COPS: que se define a partir de las categorías y variables como sigue en la Tabla 3: En la Tabla 3, Competencias personales son el conjunto de habilidades y conocimiento para el desarrollo organizacional; Adaptación es la capacidad de ajustarse a las necesidades de los clientes y el medio empresarial; Relaciones sociales se refiere al modo de cooperar e interactuar con las demás personas de la empresa; Técnicas COPs.son lasherramientas y prácticas de colaboración desde internet y redes sociales

Tabla 2. Categorías y variables de la dimensión Uso intensivo 


\begin{tabular}{|c|c|c|}
\hline Categoría & Variable & Fundamentación de autores \\
\hline \multirow{3}{*}{$\begin{array}{l}\text { Procesos } \\
\text { estratégicos }\end{array}$} & Direccionamiento & \multirow{15}{*}{$\begin{array}{l}\text { Tang et al. (2010) } \\
\text { Yang (2010) } \\
\text { Du et al. (2011) } \\
\text { Hsu et al. (2011) } \\
\text { Torresa et al. (2011) } \\
\text { Hong et al. (2012) } \\
\text { Shih- et al. (2012) } \\
\text { Verhagen et al. (2012) } \\
\text { Larsen y Olaisen, (2013) }\end{array}$} \\
\hline & Planeación & \\
\hline & Información y comunicación & \\
\hline \multirow{5}{*}{$\begin{array}{l}\text { Procesos } \\
\text { misionales }\end{array}$} & Requerimientos & \\
\hline & Diseño & \\
\hline & Construcción & \\
\hline & Pruebas & \\
\hline & Mantenimiento y soporte & \\
\hline \multirow{7}{*}{$\begin{array}{l}\text { Procesos de } \\
\text { apoyo }\end{array}$} & Gestión financiera & \\
\hline & Gestión del talento humano & \\
\hline & Comercialización y marketing & \\
\hline & Gestión Tecnológica & \\
\hline & Gestión jurídica y legal & \\
\hline & Gestión de proyectos & \\
\hline & Gestión de grupos de interés & \\
\hline
\end{tabular}

Tabla 3. Categorías y variables de la dimensión Comunidades de práctica

\begin{tabular}{|c|c|c|}
\hline Categoría & Variable & Fundamentación de autores \\
\hline \multirow{2}{*}{$\begin{array}{l}\text { Competencias } \\
\text { personales }\end{array}$} & Liderazgo & \multirow{9}{*}{$\begin{array}{l}\text { Camisón y Forés, (2011), ( } \\
\text { Jeon et al. (2011), ( } \\
\text { Siddike e Islam, (2011), ( } \\
\text { García et al.(2012), ( } \\
\text { Patel et al.(2012), ( } \\
\text { Priegue y Leiva, (2012) y ( } \\
\text { Liberona y Ruiz, (2013) }\end{array}$} \\
\hline & Potencial creativo & \\
\hline \multirow{2}{*}{ Adaptación } & Resolución de problemas & \\
\hline & Pensamiento & \\
\hline \multirow{3}{*}{$\begin{array}{c}\text { Relaciones } \\
\text { sociales }\end{array}$} & Comunicación & \\
\hline & Trabajo en equipo & \\
\hline & $\begin{array}{l}\text { Gestión de relaciones con los } \\
\text { stakeholders }\end{array}$ & \\
\hline \multirow{2}{*}{ Técnicas COPS } & Gestión de proyectos & \\
\hline & Uso de tecnologías digitales & \\
\hline
\end{tabular}

Una vez establecida la validación del modelo, en cuanto a dimensiones, categorías y variables, se definió una escala o rango de los resultados encontrados, para indicarle a cada empresa, cuál su estado real de la gestión del conocimiento. Para el efecto se hizo una revisión de los diversos modelos de madurez de evaluación de gestión del conocimiento, según: (Kulkarni y Freeze, 2004), (Robinson, et. al., 2006), (Hefke, et al., 2007), (Nove, 2009) desde el modelo de madurez (Zhao, et al., 2012) e igualmente (Jiankang, et al., 2011), realizaron una comparación de los aspectos esenciales de los niveles de madurez de algunos modelos de gestión del conocimiento. Dicha revisión permitió coincidir en el uso de cuatro escalas y una valoración de uno a 100.

\section{MATERIALES Y MÉTODOS}

Con los constructos definidos y con las variables, se continúa con la fase de diseño de la muestra, lo que supone dos cosas: 1) delimitación de las unidades de observación, y 2) la definición de las características de la muestra.

\section{Delimitación de las unidades de observación}

Se pretende observar la realidad de la GC al interior de las empresas y en ese sentido el ámbito de estudio elegido comprende a las PYMES del sector TI del triángulo del café que se dedican al desarrollo de software, la cuales aproximadamente son 150, distribuidas porcentualmente en fracciones iguales entre las ciudades de Manizales, Pereira y Armenia.

Características de la muestra 
Dado que la población es pequeña se procedió a validar el modelo en todas las PYMES, de las cuales se logró que 110 se vincularan a este proceso. Ver en https://drive.google.com/a/ucaldas.edu.co/file/d/ 0BzMqd65-Rck5NW01b0EtcFdIUWc/edit?usp=sharing.

Escala de medida: para la estructuración del modelo se formularon preguntas en escala Likert, las cuales se calificaron de 1 a 5 , donde 1 , se está en desacuerdo o no realizado, 2, realizado parcialmente, 3, realizado en intervalos de tiempo, 4 , realizado con regularidad y 5 realizado completamente.

\section{Análisis de los datos}

Basados en los planteamientos de Hair et al. (2007), frente a la evaluación de constructos teóricos, se consideran las siguientes herramientas:

Análisis de fiabilidad: la fiabilidad es el grado de consistencia entre las múltiples medidas de una variable. El objetivo es asegurar que las respuestas no varían demasiado a lo largo de periodos temporales por lo que una medida tomada en cualquier momento del tiempo es certera. Análisis de correlación: el análisis de correlación implica correlacionar simultáneamente varias variables métricas dependientes y varias variables métricas independientes. Análisis factorial: el análisis factorial proporciona una visión directa de las interrelaciones entre las variables o los encuestados y un apoyo empírico para abordar las cuestiones conceptuales que tienen relación con la estructura subyacente de los datos. También juega un papel complementario importante con otras técnicas multivariantes mediante el resumen y la reducción de los datos.

\section{RESULTADOS Y DISCUSIÓN}

\section{Análisis de correlaciones}

Con base en el constructo inicial, se procede entonces a realizar el análisis de correlaciones por variables. Los resultados se pueden observar en https://drive.google.com/file/d/0BzMqd65-Rck5QzA1RF90Q01GeVU/ edit?usp=sharing. Los resultados indican la correlación entre las variables.

\section{Análisis de fiabilidad}

Se procede a evaluar la fiabilidad y uniformidad, con el alfa de Cronbach, considerando la evaluación de los indicadores de cada variable. Los resultados se pueden observar en la tabla 4:

Tabla 4. Resultados de la evaluación de las variables del modelo

\begin{tabular}{lc}
\hline \multicolumn{1}{c}{ Categoría } & Alfa de cronbach \\
\hline Ciclo de vida & 0,906 \\
\hline Tecnologías digitales & 0,789 \\
\hline Cultura organizacional & 0,802 \\
\hline Procesos estratégicos & 0,874 \\
\hline Procesos misionales & 0,951 \\
\hline Procesos de apoyo & 0,824 \\
\hline Competencias personales & 0,775 \\
\hline Relaciones sociales & 0,827 \\
\hline
\end{tabular}

Los resultados ratifican que el constructo es fiable.

\section{Análisis factorial confirmatorio}

Para validar aún más el modelo se procede a realizar el análisis factorial confirmatorio, que genera los resultados de la tabla 5:

Se ratifica la fiabilidad del modelo. El modelo final, es el de la tabla 6:

Se procede entonces a realizar el análisis de convergencia, divergencia y fiabilidad del modelo, cuyos resultados se pueden observar en la tabla 7 y ratifican la validación del mismo.

Tabla 5. Resultados del AFC de las variables del modelo 


\begin{tabular}{|c|c|c|}
\hline Categoría & Variables & $\%$ Acum. varianza \\
\hline Ciclo de vida & Identificar, generar, retener, compartir y aplicar & 72,6 \\
\hline Tecnologías digitales & Básicas, métodos, tecnologías de conocimiento & 70,5 \\
\hline Cultura organizacional & Personas, narrativas, sitio de trabajo & 71,8 \\
\hline Procesos estratégicos & Planeación, información y comunicación & 89,2 \\
\hline Procesos misionales & Requerimientos y diseño, construcción, pruebas y mantenimiento & 95,3 \\
\hline Procesos de apoyo & $\begin{array}{l}\text { Gestión financiera, comercialización y marketing, gestión tecnológica, } \\
\text { gestión jurídica y legal y gestión de grupos de interés. }\end{array}$ & 59 \\
\hline Competencias personales & Liderazgo y potencial creativo & 85,6 \\
\hline Relaciones sociales & Comunicación y trabajo en equipo. & 85,3 \\
\hline
\end{tabular}

Tabla 6. Modelo de evaluación de GC final

\begin{tabular}{|c|c|c|}
\hline Dimensión & Categoría & Variable \\
\hline \multirow{11}{*}{ Infraestructura } & \multirow{5}{*}{ Ciclo de vida } & Identificar \\
\hline & & Generar \\
\hline & & Retener \\
\hline & & Compartir \\
\hline & & Aplicar \\
\hline & \multirow{3}{*}{ Tecnologías digitales } & Básicas \\
\hline & & Métodos \\
\hline & & Tecnologías de conocimiento \\
\hline & \multirow{3}{*}{ Cultura organizacional } & Personas \\
\hline & & Narrativa \\
\hline & & Sitio de trabajo \\
\hline \multirow{9}{*}{ Uso intensivo } & \multirow{2}{*}{ Procesos estratégicos } & Planeación \\
\hline & & Información y comunicación \\
\hline & \multirow{2}{*}{ Procesos misionales } & Requerimientos y diseño \\
\hline & & Construcción, pruebas y mantenimiento \\
\hline & \multirow{5}{*}{ Procesos de apoyo } & Gestión financiera \\
\hline & & Comercialización y marketing \\
\hline & & Gestión tecnológica \\
\hline & & Gestión jurídica y legal \\
\hline & & Gestión de grupos de interés \\
\hline \multirow{4}{*}{ COPS } & \multirow{2}{*}{ Competencias personales } & Liderazgo \\
\hline & & Potencial creativo \\
\hline & \multirow{2}{*}{ Relaciones sociales } & Comunicación \\
\hline & & Trabajo en equipo \\
\hline
\end{tabular}

Tabla 7. Análisis de Convergencia, Divergencia y Fiabilidad

\begin{tabular}{|c|c|c|c|c|c|c|c|c|c|c|c|c|c|}
\hline Dimensiones & & $\mathrm{CR}$ & AVE & MSV & ASV & DIM1 & DIM2 & DIM3 & DIM4 & DIM5 & DIM6 & DIM7 & DIM8 \\
\hline $\begin{array}{l}\text { Competencias } \\
\text { personales }\end{array}$ & DIM1 & 0,81 & 0,68 & 0,70 & 0,45 & 0,82 & & & & & & & \\
\hline ciclo de vida & DIM2 & 0,86 & 0,75 & 0,61 & 0,48 & 0,69 & 0,87 & & & & & & \\
\hline TICs & DIM3 & 0,83 & 0,62 & 0,46 & 0,40 & 0,51 & 0,68 & 0,79 & & & & & \\
\hline $\begin{array}{l}\text { Cultura } \\
\text { organizacional }\end{array}$ & DIM4 & 0,85 & 0,73 & 0,70 & 0,47 & 0,84 & 0,69 & 0,62 & 0,86 & & & & \\
\hline $\begin{array}{l}\text { Procesos } \\
\text { estratégicos }\end{array}$ & DIM5 & 0,91 & 0,84 & 0,58 & 0,48 & 0,62 & 0,75 & 0,67 & 0,76 & 0,92 & & & \\
\hline $\begin{array}{l}\text { Procesos } \\
\text { misionales }\end{array}$ & DIM6 & 0,97 & 0,93 & 0,42 & 0,34 & 0,51 & 0,57 & 0,65 & 0,51 & 0,53 & 0,97 & & \\
\hline $\begin{array}{l}\text { Procesos de } \\
\text { apoyo }\end{array}$ & DIM7 & 0,88 & 0,70 & 0,52 & 0,41 & 0,63 & 0,65 & 0,64 & 0,57 & 0,72 & 0,64 & 0,84 & \\
\hline $\begin{array}{l}\text { Relaciones } \\
\text { sociales }\end{array}$ & DIM8 & 0,84 & 0,72 & 0,64 & 0,52 & 0,80 & 0,78 & 0,63 & 0,75 & 0,76 & 0,65 & 0,63 & 0,85 \\
\hline
\end{tabular}

Escala de valoración 
Se definió una escala o rango de los resultados encontrados, para indicarle a cada empresa, su estado en cuanto a la gestión del conocimiento, la cual se basa en un factor de saturación, considerando la información recolectada, es decir se convierte la numeración inicial de 1 a 5 a 1 a 100.

Los rangos de la puntuación quedarían así:

Rango 1, valores entre cero y 25, correspondientes al nivel 1, inicial: no está estructurada la gestión del conocimiento.

Rango 2, valores entre 26 y 50, correspondientes al nivel 2, reactivo: se está buscando el conocimiento Rango 3, valores entre 51 y 75, correspondientes al nivel 3, definido: se está construyendo el conocimiento Rango 4, valores entre 76 y 100, correspondientes al nivel 4, organizado: se está transfiriendo el conocimiento

\section{Aplicación del modelo a una PYME del sector TI del eje cafetero}

A manera de lustración, se aplicó el modelo con un ingeniero de desarrollo junior de una de las empresas medianas de desarrollo de software de la ciudad de Manizales y los resultados que se generaron fueron los siguientes (ver figura 2):

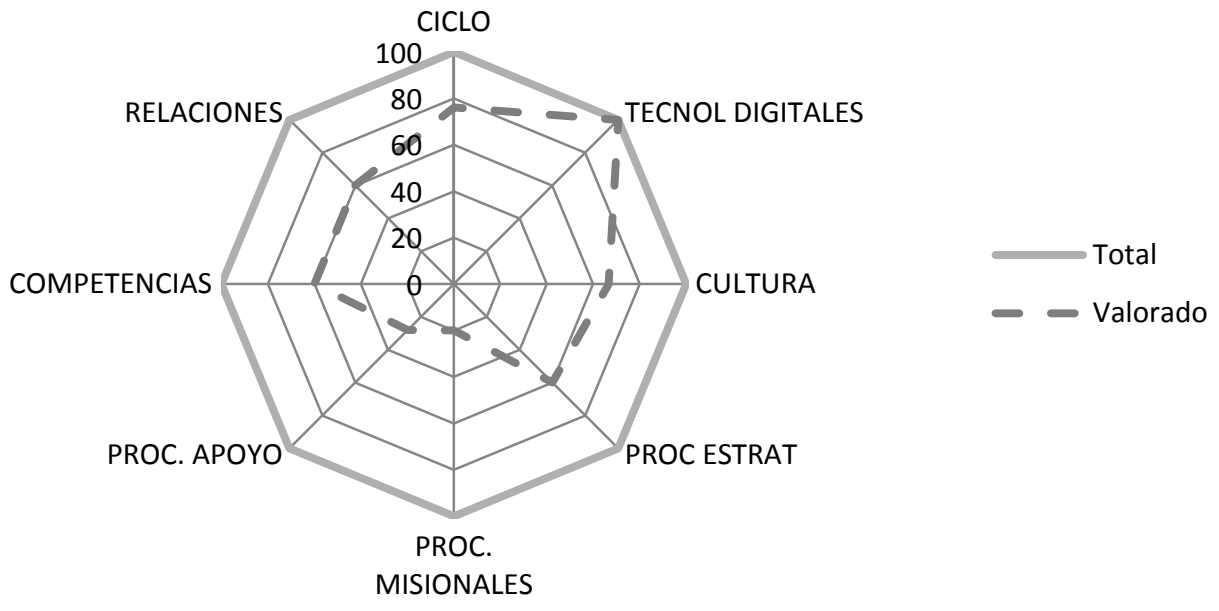

Fig. 2: Resultados de variables evaluadas de la empresa

Dichos resultados destacan de las categorías evaluadas una muy baja puntuación en procesos misionales, en procesos de apoyo y en procesos estratégicos, lo que implica la necesidad de desarrollar planes, programas y proyectos para incorporar la GC en éstas. Una puntuación alta en ciclo y cultura, lo que muestra como el ciclo de conocimiento y la cultura de GC pueden ser apropiados por los empleados de la organización y una puntuación muy alta en tecnologías digitales, propias de este tipo de compañías.

Ya en lo relacionado con el estado de la GC, según la escala propuestas, se observan los resultados en la figura 3.

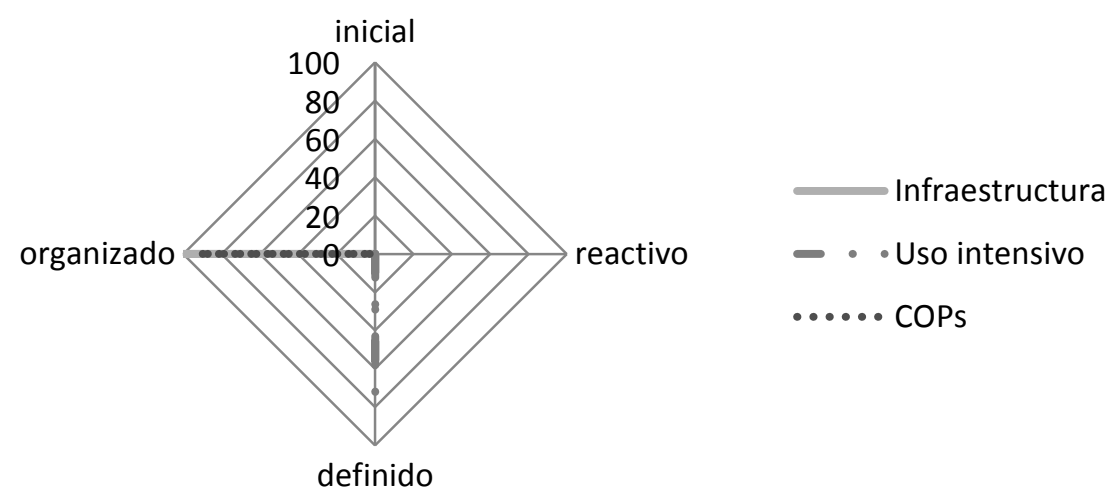

Fig. 3: Estado de la GC en la empresa 
Los resultados muestran lo siguiente: en infraestructura el estado es organizado, es decir, en la cultura se practica compartir los activos de conocimiento; las actividades de gestión del conocimiento hacen parte de los flujos de trabajo, existen repositorios de conocimiento y una taxonomía del know how organizacional. Las tecnologías están definidas, es decir, los métodos y herramientas digitales de GC son de uso común, existen mecanismos sistemáticos para facilitar actividades de gestión del conocimiento. En el ciclo se incorpora compartir conocimiento y se definen acciones para identificar y retener el conocimiento. En lo concerniente al uso intensivo el estado es definido, es decir se institucionaliza el uso del conocimiento en los procesos organizacionales y se gestiona la base de conocimiento organizacional. Ya en lo referido a COPs están en estado organizado, es decir, existe estrategia construida, hay liderazgo colaborativo, se tienen definidos los roles, se crean contenidos, hay gobernanza flexible y mezclada con los grupos de interés.

Los resultados del desarrollo del modelo de evaluación de la gestión del conocimiento en las empresas del sector TI del eje cafetero, permitiría igualmente contrastar hipótesis alrededor de: las tecnologías digitales, la cultura organizacional y el ciclo de vida para la GC; las relaciones sociales y las competencias personales para la GC. El ciclo de conocimiento, las tecnologías digitales y los procesos estratégicos para la GC. La cultura organizacional, los procesos de apoyo y las competencias personales para la GC. La cultura organizacional, los procesos estratégicos y las relaciones sociales para la GC y los procesos de apoyo, la cultura y las tecnologías digitales para la GC, entre otros.

\section{CONCLUSIONES}

Las métricas de valoración validadas para las empresas de desarrollo de software del sector TI del eje cafetero, se consideran una innovación en cuanto a la aplicación de la gestión por procesos y la integralidad de la evaluación, toda vez que no existe hasta el momento un modelo parecido.

Una de las limitaciones del estudio desde el punto de vista estadístico fue el no contar con la información del total de la población, lo que puede limitar en algún momento la generalización de los resultados a otros ámbitos geográficos o sectoriales.

Un constructo teórico debe tener como fundamento los marcos de referencia establecidos desde la revisión bibliográfica, en este caso se tuvieron en cuenta modelos de gestión del conocimiento, modelos de evaluación de gestión del conocimiento y otros sobre la evaluación de gestión del conocimiento, en este caso, la mayoría de los elementos teóricos revisados, consideran las variables presentadas, integradas al planteamiento de las categorías de infraestructura, procesos y comunidades de práctica, que se presentaron.

Para asegurar que dicho modelo realmente se pudiera aplicar a un contexto, en este caso las PYMES del sector TI, fue necesario realizar una validación estadística, desde las herramientas tradicionales y desde el análisis factorial confirmatorio, para demostrar la eficacia modelo teórico propuesto.

Dada la importancia de la GC en el mundo empresarial actual, es definitivo evaluar el estado de su desarrollo en las empresas, pero también establecer, planes programas y proyectos que permitan llegar al estado ideal.

\section{REFERENCIAS}

Barney, J. Firm Resources and sustained competitive advantage. Journal of management, 99-100. (1991)

Benavides, C.Tecnología, innovación y empresa. Madrid: Ediciones Piramide. (1998)

Cameron, K., y Quinn, R. Diagnosing and Changing Organizational Culture. Addison- Wesley, Series on Organization. (1999)

Camisón, C., y Forés, B. Knowledge creation and absorptive capacity: The effect of intra-district shared competences. Scandinavian Journal of Management (22), 66-86. (2011)

Castells, M. De la función de producción agregada a la frontera de posibilidades de producción: productividad, tecnología y crecimiento económico en la era de la información. Barcelona: Real Academica de Ciencias Economicas y Financieras. (2006) 
CEN-3. European Guide to good Practice in Knowledge Management - Part 3: SME Implementation. Recuperado el 01 de 05 de 2013, de European comittee for standarization: http://www.cen.eu/cen/Sectors/Sectors/ISSS/CWAdownload/Pages/Knowledge\%20Management.aspx. (2004)

Chang, T.-H., y Wang, T.-C. Using the fuzzy multi-criteria decision making approach for measuring the possibility of successful knowledge management. Information Sciences (179), 355-370. (2009)

Chen, T. A multiple-layer knowledge management system framework considering user knowledge privileges. International Journal of software engineering an knowledge engineering, 361-387. (2008)

Chen, M.-Y., y Chen, C.-C. Options analysis and knowledge management: Implications for theory and practice. Information Sciences (181), 3861-3877. (2011)

Condo, A. Desarrollo de clústeres competitivos. Centro Latinoamericano para la Competitividad y el Desarrollo Sostenible. Recuperado el 25 de 11 de 2011, de CLACDS:

http://www.conindustria.org/clusterscompetitivos. (2001)

DelMoral, A., Pazos, J., Rodríguez, E., Rodríguez, P., y Suarez, S. Gestión del Conocimiento (1ª edición ed.). Madrid: Thomson. (2007)

Dosi, G. T. Toward a theory of corporate coherence: preliminary remarks. Technology and Enterprise in a Historical Perspective. (1992)

Du, M., Qiu, F., y Xu, W. Construction of Enterprises' Financial Knowledge Management System (EFKMS). Procedia Environmental Sciences(11), 1240 - 1244. (2011)

Eftekharzade, S., y Mohammadi, B. The Presentation of a Suitable Model for Creating Knowledge Management in Educational Institutes (Higher Education). Procedia - Social and Behavioral Sciences(29), 1001 - 1011. (2011)

Folta, T. C. Geographic cluster size and firm performance. Journal of Business Venturing, 217-242. (2006)

Gallagher, C. New firm starts, growth and clusters in East London". Rising East. The Journal of East London Studies, 25. (2001)

García-Barriocanal, E., Sicilia, M.-A., y Sánchez-Alonso, S. Computing with competencies: Modelling organizational capacities. Expert Systems with Applications(39), 12310-12318. (2012)

Hair, J., Anderson, R., Tatham, R., y Black, W. Análisis multivariante. Madrid: Pearson. (2007)

Hefke, M., Kleiner, F., y Storkenmaier, A. Retaining Knowledge Management Maturity Models: An Ontologybased Approach. I-KNOW '07, (págs. 1-8). Graz, Austria. (2007)

Hong, A., Yip, M., Din, S., y Bakarb, N. Integrated Knowledge Management Strategy: A Preliminary Literature Review. Procedia - Social and Behavioral Sciences(57), 209 - 214. (2012)

Hsu, J., Liang, T., Wu, S., Klein, G., y Jiang, J. Promoting the integration of users and developers to achieve a collective mind through the screening of information system projects. International Journal of Project Management(29), 514-524. (2011)

Hsun, Y., Chou, S.-C. T., y Tzeng, G.-H. Knowledge management adoption and assessment for SMEs by a novel MCDM approach. Decision Support Systems, 270-291. (2011)

Irani, Z., Sharif, A., Mustafa, M., y Love, P. Visualising a knowledge mapping of information systems investment evaluation Expert Systems with Applications. Expert Systems with Applications(41), 105-125. (2014)

Jeon, S.-H., Kim, Y.-G., y Koh, J. Individual, social, and organizational contexts for active knowledge sharing in communities of practice. Expert Systems with Applications(38), 12423-12431. (2011) 
Jennex, M. E., y Olfman, L. Assessing Knowledge Management Success/Effectiveness Models. Proceedings of the 37th Hawaii International Conference on System Sciences, (págs. 1-10). (2004)

Jiankang, W., Jiuling, X., Qianwen, L., y Kun, L. Knowledge Management Maturity Models:A Systemic Comparison. International Conference on Information Management, Innovation Management and Industrial Engineering, (págs. 606-609). (2011)

Joyanes, L. La computación en nube (cloud computing): el nuevo paradigma tecnológico para empresas y organizaciones en la sociedad del conocimiento. Revista de las Facultades de Derecho y Ciencias Económicas y Empresariales, 95-111. (2009)

Kaplan, R., y Norton, D. Using the Balanced Scorecard as a Strategic Management System. Harvard Business Review, 76. (1996)

Karadsheh, L., Mansour, E., Alhawari, S., Azar, G., y El-Bathy, N. A Theoretical Framework for Knowledge Management Process: Towards Improving Knowledge Performance. Communications of the IBIMA, 7, 6779. (2009)

Kruger, C., y Johnson, R. Information management as an enabler of knowledge management maturity: $A$ South African perspective. International Journal of Information Management(30), 57-67. (2010).

Kulkarni, U., y Freeze, R. Twenty-Fifth International Conference on Information Systems. Recuperado el 05 de 01 de 2014, de

http://pdf.aminer.org/000/326/698/development_and_validation_of_a_knowledge_management_capability_a ssessment_model.pdf. (2004)

Larsen, T., y Olaisen, J. Innovating strategically in information and knowledge management: Applications of organizational behavior theory. International Journal of Information Management(33), 764- 774. (2013)

León Santos, M., y Ponjuán Dante, G. Propuesta de un modelo de medición para los procesos de la gestión del conocimiento en organizaciones de información. Interamericana de Bibliotecología, 34, 87-103. (2011)

Liberona, D., y Ruiz, M. Análisis de la implementación de programas de gestión del conocimiento en las empresas chilenas. Estudios gerenciales(29), 151-160. (2013)

Limone, A., y Bastidas, L. La empresa y la gestión del conocimiento en el contexto de la revolución cibernética. Valparaiso, Chile: Pontificia Universidad Católica de Valparaíso. Facultad de Ciencias Económicas y Administrativas. (2003)

Lopez, Marcelo; Hernandez, Albeiro y Marulanda, Carlos E. Procesos y Prácticas de Gestión del Conocimiento en Cadenas Productivas de Colombia. Inf. tecnol. [online]. vol.25, n.3, pp. 125-134. ISSN 0718-0764. (2014)

López, M. Ciudadanía Digital, un modelo de implantación en la región de Manizales y Caldas, Colombia. Madrid: Universidad pontificia de Salamanca. (2010)

Mueller, J. A specific knowledge culture: Cultural antecedents for knowledge sharing between project teams. European Management Journal, 1-13. (2013)

Nove-Eka, V.-A. Knowledge Management Implementation and Its Maturity Level. Media Indonesian Journal of Social Sciences, 1 (1), 1-13. (2009)

Patel, H., Pettitt, M., y Wilson, J. R. Factors of collaborative working: A framework for a collaboration model. Applied Ergonomics(43), 1-26. (2012)

Pawlowsk, J., y Bick, M. The Global Knowledge Management Framework: Towards a Theory for Knowledge Management in Globally Distributed Settings. Recuperado el 01 de 08 de 2013, de The Electronic Journal of Knowledge: www.ejkm.com. (2012).

Porter, M. Clusters of Innovation: Regional Foundations of U.S. Competitiveness. Council of Competitiveness (Online). Obtenido de http://www.compete. org/pdf/national_report.pdf. (2001) 
Priegue, D., y Leiva, J. Las competencias interculturales en la sociedad del conocimiento: reflexiones y análisis pedagógico. Revista electrónica de tecnología educativa(40), 1-12. (2012)

Proexport. Software y Servicios de TI. Bogotá: Ministerio de Comercio exterior. (2011)

Riesco, M. El negocio es el conocimiento. Madrid: Díaz de Santos. (2006)

Robinson, H.S., C.J. Anumba, P.M. Carrillo y A.M. Al-Ghassani. Knowledge management. Loughborough university. Recuperado el 05 de 01 de 2014, de https://dspace.lboro.ac.uk/. (2006)

Sanabria, S., Morales, M., y Arias, M., Conocimiento, innovación y competitividad en aglomeraciones empresariales. Facultad de Ciencias económicas, 19-53. (2010)

Sánchez, A. Modelo para la medición del capital intelectual de territorios insulares: una aplicación al caso de Gran Canaria. España. Tesis doctoral de la Universidad de las Palmas de Gran Canaria. , 75. (2003)

Sánchez, J. Algunas aproximaciones al problema de financiamiento en las PYMES de Colombia. Ciencia y técninca , 321-324. (2007)

Shapira, P., Youtie, J., Yogeesvaran, K., y Jaafar, Z., Knowledge economy measurement: Methods, results and insights the Malaysian Knowledge Content Study. Research Policy 35, 1522-1537. (2006)

Shih-Chieh, J., Lin, T.-C., Zheng, G.-T., \& Hung, Y.-W. Users as knowledge co-producers in the information system development project. International Journal of Project Management(30), 27-36. (2012)

Siddike, A., e Islam, S. Exploring the competencies of information professionals for knowledge management in the information institutions of Bangladesh. The International Information \& Library Review(43), 130 - 136. (2011)

Steinfield, C., Scupola, A., y López-Nicolás, C. Social capital, ICT use and company performance: Findings from the Medicon Valley Biotech Cluster. Technological Forecasting \& Social Change(77), 1156-1166. (2010)

Sulayman, M., Urquhart, C., Mendes, E., y Seidel, S. Software process improvement success factors for small and medium Web companies: A qualitative study. Information and Software Technology, 479-500. (2012)

Tallman, S. J. Knowledge, clusters, and competitive advantage. Academy of Management Review, 258-271. (2004)

Tang, A., Avgeriou, P., Jansen, A., Capilla, R., y Ali, M. A comparative study of architecture knowledge management tools. The Journal of Systems and Software(83), 352-370. (2010)

Torresa, T., Pierozzi, I., Rodrigues, N., y Castro, A. Knowledge management and communication in Brazilian agricultural research: An integrated procedural approach. International Journal of Information Management(31), 121-127. (2011)

Vaccaro, A., Parente, R., y Veloso, F. M. Knowledge Management Tools, Inter-Organizational Relationships, Innovation and Firm Performance. Technological Forecasting \& Social Change(77), 1076-1089. (2010)

Verhagen, W., Bermell-Garcia, P., Van, R., y Curran, R. A critical review of Knowledge-Based Engineering: An identification of research challenges. Advanced Engineering Informatics(26), 5-15. (2012)

Wen, Y.-F. An effectiveness measurement model for knowledge management. Knowledge-Based Systems, 22, 363-367. (2009)

Yang, J. The knowledge management strategy and its effect on firm performance: A contingency analysis. Production Economics(125), 215-223. (2010)

Zhao, J., Ordóñez de Pablos, P., y Qi, Z. Enterprise knowledge management model based on China's practice and case study. Computers in Human Behavior (28), 324-330. (2012) 